\title{
ASPECTOS SENSORIAIS E FÍSICO-QUÍMICOS DE "IOGURTES" DE SOJ A COM ESPESSANTES/ ESTABILIZANTES A BASE DE FÉCULA DE INHAME (Dioscorea alata), AMIDO MODIFICADO E GELATINA
}

\author{
GABRIELAPAULINO PANHONI MANZANO* \\ ERICA REGINA DAIUTO** \\ NATÁLIASOARES JANZANTTI*** \\ ELIZEU ANTONIO ROSSI***
}

\begin{abstract}
Este estudo teve como objetivo avaliar o efeito da fécula de inhame, amido modificado da Cargill-Brasil (Amidomax $4800^{\circledR}$ ) e gelatina da Gelita-Brasil (GELLAC $^{\circledR}$ ) como espessantes/estabilizantes em diferentes proporções e combinações em "iogurte" de soja fermentado com Enterococcus faecium e Lactobacillus helveticus ssp jugurti. Dez formulações de "iogurte" de soja contendo os diferentes espessantes/estabilizantes, sempre totalizando $0,5 \%$ em relação à formulação final, foram analisadas em termos sensoriais e físico-químicos. Com base nos resultados observados concluiu-se que sob o ponto de vista sensorial, o produto mais adequado foi processado apenas com gelatina na concentração de $0,5 \%$. Esse produto também apresentou os melhores resultados físico-químicos em relação à consistência, sinérese e capacidade de retenção de água. No entanto, a gelatina utilizada isoladamente provocou aumento no tempo de fermentação do "iogurte" de soja.
\end{abstract}

PALAVRAS-CHAVE: IOGURTE DE SOJA; INHAME; AMIDO MODIFICADO; GELATINA; ANÁLISE SENSORIAL; ANÁLISES FISÍCO-QUÍMICAS.

* $\quad$ Mestre em Alimentos, Departamento de Alimentos e Nutrição, Faculdade de Ciências Farmacêuticas, Universidade Estadual Paulista (UNESP), Araraquara, SP (e-mail: gpanhoni@hotmail.com).

** Pós-doutoranda em Horticultura, Professora, Curso de Especialização em Ciência e Tecnologia de Alimentos, Faculdade de Ciências Agronômicas, UNESP, Botucatu, SP (e-mail: erdaiuto@yahoo.com.br).

*** Pós-doutoranda em Alimentos, Departamento de Alimentos e Nutrição, Faculdade de Ciências Farmacêuticas, UNESP, Araraquara, SP (e-mail: natalia@fcfar.unesp.br).

**** Professor Adjunto, Departamento de Alimentos e Nutrição, Faculdade de Ciências Farmacêuticas, UNESP, Araraquara, SP (e-mail: rossiea@fcfar.unesp.br). 


\section{INTRODUÇÃO}

A soja é uma leguminosa conhecida por suas propriedades funcionais benéficas à saúde humana. O "iogurte" de soja obtido a partir do extrato hidrossolúvel da soja, produto similar ao iogurte, apresenta boa aceitabilidade, custo reduzido e mantém preservadas as características nutricionais e terapêuticas apresentadas pelos produtos fermentados convencionais (ROSSI, REDDY e SILVA, 1984). Esse produto fermentado com cepa específica de Enterococcus faecium revelou vários efeitos benéficos já constatados cientificamente, dentre os quais a redução dos níveis séricos de colesterol, estímulo do sistema imunológico e prevenção da osteoporose (SHIGUEMOTO et al., 2007; BEDANI, 2005; ROSSI et al., 2003; VENDRAMINI, 2002; ROSSI et al., 2000). Trata-se de produto com boa aceitabilidade sensorial, mas com parâmetros reológicos ainda não totalmente adequados, especialmente no tocante à consistência.

As gomas e a gelatina são utilizadas como espessantes/estabilizantes em iogurtes convencionais, conferindo-lhes melhor consistência e redução da sinérese. No entanto, não foi observado o mesmo efeito no "iogurte" de soja.

Quando se trata de iogurte, a textura e o corpo são tão importantes quanto o próprio sabor. Firmeza adequada e ausência de sinérese são essenciais para se obter produto de alta qualidade. Estudos já foram realizados com o objetivo de melhorar a estabilidade e a consistência do "iogurte" de soja, empregando misturas de várias gomas e gelatina em proporções diversas (ROSSI et al., 1990). O uso de amido, produto bastante difundido e proveniente de matérias-primas tipicamente brasileiras, no entanto, ainda não foi estudado em iogurtes.Vale ressaltar que o amido, juntamente com outros hidrocolóides, pode melhorar as características de determinados alimentos em relação a sinérese e a consistência (MALI et al., 2003). Dentre os amidos nativos utilizados pela indústria de alimentos, os principais são provenientes do milho, da mandioca, da batata doce, do trigo e do arroz.

Pesquisas vêm sendo realizadas visando a caracterização de féculas de tuberosas amiláceas para aplicação em alimentos (CEREDA et al., 2001). Estudos relacionados com as propriedades funcionais também foram efetuados (GUERREIRO, 2002; DAIUTO, 2005), mas sem a devida avaliação em condições reais de aplicação na indústria de alimentos.

A fécula de inhame (Dioscorea alata) apresenta propriedades para aplicação em alimentos, porém sua extração é normalmente dificultada pela presença de mucilagem nos tubérculos. Por essa razão DAIUTO e CEREDA (2003) propuzeram metodologia visando facilitar o processo de extração utilizando mistura de oxalato de amônio e ácido oxálico.

Considerando o custo relativamente alto da gelatina, a fécula de inhame associada ou não com outros espessantes/estabilizantes pode representar alternativa econômica e tecnologicamente viável para melhorar as propriedades reológicas do "iogurte" de soja.

Este trabalho teve como objetivo avaliar o comportamento de fécula de inhame, amido modificado e gelatina em diferentes combinações e proporções em "iogurte" a base de extrato aquoso de soja, fermentado com Enterococcus faecium e Lactobacillus helveticus ssp jugurti, bem como verificar interferências sensoriais e físico-químicas nos produtos obtidos.

\section{MATERIAL E MÉTODOS}

Os experimentos foram realizados no Laboratório de Tecnologia de Alimentos da Faculdade de Ciências Farmacêuticas da Universidade Estadual Paulista (UNESP), campus de Araraquara.

Estudos prévios (dados não-publicados) foram realizados para selecionar alguns espessantes/ estabilizantes utilizados no "iogurte" de soja, fermentado com Enterococcus faecium e Lactobacillus helveticus ssp jugurti, tais como: farinha e fécula de inhame, amidos modificados obtidos da CargillBrasil (Amidomax $4800^{\circledR}$ e 5500 ${ }^{\circledR}$ ) e da National-Brasil (National $465^{\circledR}$ ) e gelatina (GEL-LAC ${ }^{\circledast}$ ) da empresa 
Gelita-Brasil. Com base nesses resultados foram definidos os espessantes/estabilizantes a base de fécula de inhame, o amido modificado da Cargill-Brasil (Amidomax $4800^{\circledR}$ ) e a gelatina (GEL-LAC ${ }^{\circledR}$ ) da empresa Gelita-Brasil para o estudo da estabilidade e da textura do "iogurte” de soja.

\subsection{OBTENÇÃO DA FÉCULA DE INHAME}

A extração da fécula ocorreu conforme metodologia descrita por DAIUTO e CEREDA, 2003. Os tubérculos foram lavados, descascados e cortados em pedaços pequenos para posterior trituração em solução a $10 \%$ de oxalato de amônio e ácido oxálico (proporção de 1:1). Em seguida, a pasta obtida foi passada por tamises de números 100 e 200 (malhas de $150 \mu \mathrm{m}$ e $75 \mu \mathrm{m}$, respectivamente). Descartou-se o produto retido (mucilagem) e o restante foi decantado. Depois de eliminado o sobrenadante, o resíduo foi desidratado a $40^{\circ} \mathrm{C}$ para a obtenção da fécula.

Dez tipos de formulações de "iogurte" de soja foram analisados com diferentes proporções e combinações dos espessantes/estabilizantes, conforme apresentado na Tabela 1.

\section{TABELA 1 - PROPORÇÕES* E COMBINAÇÕES DOS DIFERENTES ESPESSANTESI ESTABILIZANTES UTILIZADOS NO “IOGURTE” DE SOJA}

\begin{tabular}{|c|c|c|c|c|}
\hline Tratamentos & Gelatina (\%) & $\begin{array}{c}\text { Fécula de Inhame } \\
(\%)\end{array}$ & $\begin{array}{c}\text { Amido Modificado } \\
\text { Amidomax } 4800^{\circledast}(\%)\end{array}$ & $\begin{array}{c}\text { \% Total das } \\
\text { combinações }\end{array}$ \\
\hline T1 & 0,00 & 0,50 & 0,00 & 0,50 \\
\hline T2 & 0,00 & 0,00 & 0,50 & 0,50 \\
\hline T3 & 0,00 & 0,25 & 0,25 & 0,50 \\
\hline T4 & 0,20 & 0,30 & 0,00 & 0,50 \\
\hline T5 & 0,20 & 0,00 & 0,30 & 0,50 \\
\hline T6 & 0,30 & 0,20 & 0,00 & 0,50 \\
\hline T7 & 0,50 & 0,00 & 0,00 & 0,50 \\
\hline T8 & 0,25 & 0,25 & 0,00 & 0,50 \\
\hline T9 & 0,25 & 0,00 & 0,25 & 0,50 \\
\hline T10 & 0,15 & 0,20 & 0,15 & 0,50 \\
\hline
\end{tabular}

*As quantidades de cada espessante/estabilizante enquadraram-se nos limites da região experimental, definida em ensaios preliminares com base em testes físicos e sensoriais, cujos resultados ainda não foram publicados.

\subsection{OBTENÇÃO DOS “IOGURTES” DE SOJA}

O "iogurte" foi elaborado com "leite" de soja obtido da Unidade de Produção e Desenvolvimento de Derivados de Soja (UNISOJA), UNESP de Araraquara. Usou-se a metodologia descrita por ROSSI, REDDY e SILVA (1984) com algumas alterações. Reduziu-se a quantidade de óleo de soja de $2,6 \%$ para $0,8 \%$, a de açúcar de $10 \%$ para $6 \%$ e a de leite em pó de 3,5\% para $2,5 \%$. Em substituição ao soro de leite utilizou-se $1 \%$ de lactose, sendo o inóculo convencional substituído por cultivos de Enterococcus faecium e Lactobacillus helveticus ssp jugurti na proporção de $1,5 \%(\mathrm{v} / \mathrm{v})$ de cada microrganismo em relação ao volume final do produto. O processo fermentativo foi conduzido até que o $\mathrm{pH}$ alcançasse valor entre 4,3 e 4,5. Não foram acrescentados corantes nem aromatizantes. Os diferentes espessantes/estabilizantes avaliados foram incorporados ao produto na proporção total de $0,5 \%(\mathrm{p} / \mathrm{v})$. 


\subsection{AVALIAÇÃO DOS "IOGURTES" DE SOJA}

\subsubsection{Análise Sensorial}

As formulações processadas (Tabela 1) foram avaliadas pelo teste de aceitação mediante escala hedônica estruturada de 9 pontos, cujos extremos corresponderam a "desgostei muitíssimo" (1) e gostei muitíssimo (9) (STONE e SIDEL, 1993). Oitenta julgadores não-treinados avaliaram as formulações em relação à textura, impressão global e atitude de compra. Os procedimentos e as condições ambientais da análise sensorial seguiram as recomendações de MORAES (1985).

\subsubsection{Análises Físico-químicas}

As amostras foram submetidas às determinações de tempo de fermentação (TAMIME e ROBINSON, 1985), pH (AOAC, 1996), capacidade de retenção de água (HARTE et al., 2003) e sinérese (HASSAN et al., 1996). Avaliou-se a consistência pela distância $(\mathrm{cm})$ percorrida pela amostra mantida a $5^{\circ} \mathrm{C}$, utilizando alíquotas de $80 \mathrm{~mL}$ em intervalo de tempo de 30 segundos em consistômetro de Bostwick (BOSTWICK CONSISTOMETER, [s.d]).

\subsection{ANÁLISE ESTATÍSTICA DOS RESULTADOS}

Os dados dos testes sensoriais e físico-químicos foram avaliados pela Análise de Variância (ANOVA) e teste de médias de Tukey para $p \leq 0,05$ por meio do programa SAS (1996), sendo apresentados também na forma de histogramas. Os dados do teste de aceitação e físico-químicos foram submetidos à análise de correlação de Pearson.

\section{RESULTADOS E DISCUSSÃO}

\subsection{ANÁLISE SENSORIAL}

Os resultados obtidos no teste de aceitação dos dez diferentes tipos de tratamentos (Tabela 1) não diferiram significativamente $(p \leq 0,05)$ para o atributo textura. Em relação à impressão global, as amostras 7 e 6 diferiram significativamente $(p \leq 0,05)$ entre $s i$, mas não das demais $(p \leq 0,05)$ (Tabela 2). Considerando que a formulação 6 não diferiu significativamente das demais pode-se dizer que sob o ponto de vista sensorial, todas as formulações apresentaram aceitação equivalente em termos de textura e de impressão global. Entretanto, cabe ressaltar que a formulação 7 obteve os melhores resultados para textura e impressão global em termos de valores absolutos.

JAWALEKAR et al. (1993), citados por KUMAR e MISHRA (2004), utilizaram a gelatina como espessante/estabilizante na formulação de iogurte a base de leite de vaca e de búfala. Obtiveram resultados semelhantes aos verificados para a aceitação da amostra 7 , contendo $0,5 \%$ de gelatina. KUMAR e MISHRA (2004) avaliaram as propriedades do "iogurte" de soja, fortificado com manga e formulado com diferentes espessantes/estabilizantes em relação à aceitação sensorial. Constataram que a formulação com gelatina a $0,4 \%$ apresentou os melhores resultados.

A segunda melhor formulação em termos de valores absolutos para a textura foi a de número 3 com $0,25 \%$ de cada tipo de amido (modificado e inhame), seguida pelas amostras 8 e 9 com 0,25\% de gelatina e $0,25 \%$ de amido de inhame ou modificado. Apesar da gelatina em maior porcentagem resultar em produto com melhor aceitação de textura (amostra 7), pode-se supor que produtos com amidos ou a mistura de amidos e gelatina seriam bem aceitos. 


\section{TABELA 2 - MÉDIAS DE ACEITAÇÃOํํㅁ DAS AMOSTRAS DE "IOGURTE" DE SOJA RESULTANTES DE DEZ TRATAMENTOS COM DIFERENTES PROPORÇÕES E COMBINAÇÕES DE ESPESSANTES/ESTABILIZANTES}

\begin{tabular}{ccc}
\hline \hline Amostras & Textura & Impressão Global \\
\hline $\mathbf{1}$ & $6,88^{\mathrm{a}}$ & $6,28^{\mathrm{ab}}$ \\
$\mathbf{2}$ & $6,94^{\mathrm{a}}$ & $6,58^{\mathrm{ab}}$ \\
$\mathbf{3}$ & $7,20^{\mathrm{a}}$ & $6,74^{\mathrm{ab}}$ \\
$\mathbf{4}$ & $6,82^{\mathrm{a}}$ & $6,70^{\mathrm{ab}}$ \\
$\mathbf{5}$ & $6,68^{\mathrm{a}}$ & $6,52^{\mathrm{ab}}$ \\
$\mathbf{6}$ & $6,54^{\mathrm{a}}$ & $6,14^{\mathrm{b}}$ \\
$\mathbf{7}$ & $7,34^{\mathrm{a}}$ & $7,02^{\mathrm{a}}$ \\
$\mathbf{8}$ & $7,06^{\mathrm{a}}$ & $6,66^{\mathrm{ab}}$ \\
$\mathbf{9}$ & $7,00^{\mathrm{a}}$ & $6,84^{\mathrm{ab}}$ \\
$\mathbf{1 0}$ & $6,88^{\mathrm{a}}$ & $6,42^{\mathrm{ab}}$ \\
\hline
\end{tabular}

Médias seguidas pela mesma letra na mesma coluna não diferiram entre $s i(p \leq 0,05), n=80$ julgadores.

$1=0 \%$ Gelatina $(G)+0,5 \%$ Amido de Inhame (Al) $+0 \%$ Amidomax $4800^{\circledR}\left(A 4800^{\circledR}\right) ; 2=0 \%$ Gelatina $(G)+0 \%$ Amido de Inhame $(A I)+0,5 \%$ Amidomax $4800^{\circledR}\left(A 4800^{\circledR}\right) ; 3=0 \%$ Gelatina $(G)+0,25 \%$ Amido de Inhame $(A I)+0,25 \%$ Amidomax $4800^{\circledR}\left(\mathrm{A} 4800^{\circledR}\right) ; 4=0,2 \%$ Gelatina (G) $+0,3 \%$ Amido de Inhame (AI) + 0\% Amidomax $4800^{\circledR}\left(A 4800^{\circledR}\right) ; 5=0,2 \%$ Gelatina (G) + $0 \%$ Amido de Inhame (AI) + 0,3\% Amidomax $4800^{\circledR}\left(A 4800^{\circledR}\right) ; 6=0,3 \%$ Gelatina $(G)+0,2 \%$ Amido de Inhame (AI) + 0\% Amidomax $4800^{\circledR}\left(A 4800^{\circledR}\right) ; 7=0,5 \%$ Gelatina $(G)+0 \%$ Amido de Inhame (AI) + 0\% Amidomax 4800 ${ }^{\circledR}\left(A 4800^{\circledR}\right) ; 8=0,25 \%$ Gelatina (G) + 0,25\% Amido de Inhame (Al) + 0\% Amidomax $4800^{\circledR}\left(A 4800^{\circledR}\right) ; 9=0,25 \%$ Gelatina (G) + 0\% Amido de Inhame $(\mathrm{Al})+0,25 \%$ Amidomax $4800^{\circledR}\left(\mathrm{A} 4800^{\circledR}\right) ; 10=0,15 \%$ Gelatina $(\mathrm{G})+0,2 \%$ Amido de Inhame (AI) + 0,15\% Amidomax $4800^{\circledR}$ $\left(\mathrm{A} 4800^{\circledR}\right)$.

${ }^{1}(1$ = desgostei muitíssimo; 9 = gostei muitíssimo).

A amostra 9, com $0,25 \%$ de gelatina e $0,25 \%$ de amido modificado, obteve a segunda melhor média para a impressão global, seguida pela amostra 3 com $0,25 \%$ de cada espessante/estabilizante. Novamente, pode-se considerar a hipótese de boa aceitação do produto apenas com amidos ou mistura de amidos e gelatina na sua composição.

A Figura 1 mostra a distribuição das freqüências dos valores atribuídos para textura e impressão global e, independente do espessante/estabilizante utilizado, cerca de $70 \%$ dos julgadores apresentaram respostas favoráveis (valores de 6 a 9).

Os valores obtidos em relação à atitude de compra estão representados no histograma apresentado na Figura 2.

Os resultados obtidos para a atitude de compra refletem preferência pela amostra 7, com $0,5 \%$ de gelatina, conforme verificado pelos testes de aceitação para textura e impressão global. A amostra 9 , com $0,25 \%$ de gelatina e $0,25 \%$ de amido modificado, também apresentou bons resultados confirmando os testes de aceitação para textura e impressão global. A amostra 6 , com $0,3 \%$ de gelatina e $0,2 \%$ de amido de inhame, não revelou bons resultados com relação à atitude de compra conforme indicado pelos testes de aceitação para impressão global. A amostra 1, com 0,5\% de amido de inhame, também não obteve resultados satisfatórios para a atitude de compra. Pode-se supor que o mercado daria preferência para "iogurtes" de soja com gelatina como espessante/estabilizante, seguido pelo produto com $0,25 \%$ de gelatina e $0,25 \%$ de amido modificado. O produto apenas com amido de inhame seria, provavelmente, o menos preferido. 


\section{FIGURA 1 - HISTOGRAMAS DE FREQÜÊNCIA DAS NOTAS ATRIBUÍDAS À ACEITAÇÃO DOS ATRIBUTOS SENSORIAIS DO "IOGURTE" DE SOJA}

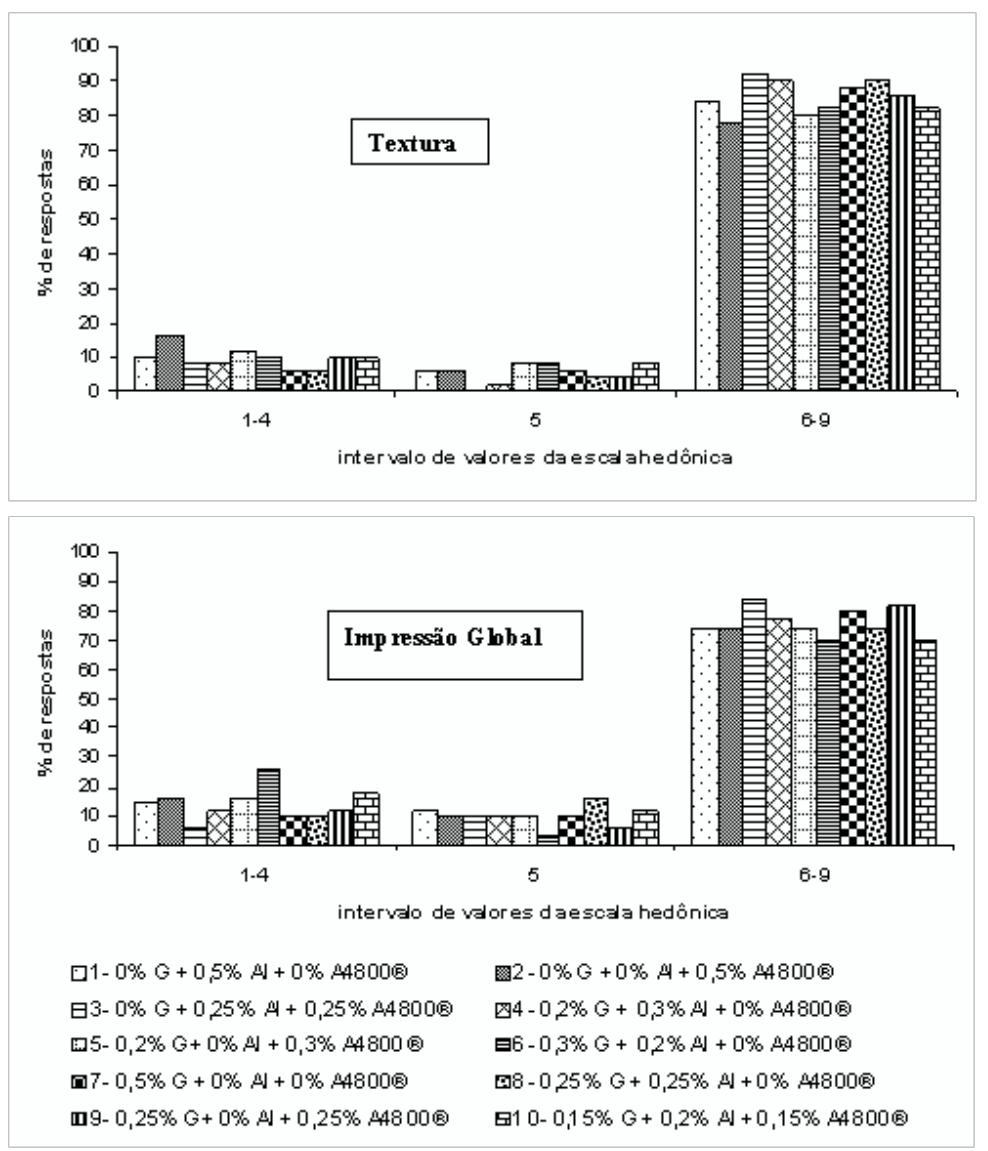

$\mathrm{G}=$ Gelatina; $\mathrm{Al}=$ Amido de Inhame; $\mathrm{A} 4800^{\circledR}=$ Amidomax $4800^{\circledR}$.

\section{FIGURA 2 - REPRESENTAÇÃO GRÁFICA DOS RESULTADOS DE ATITUDE DE COMPRA EM RELAÇÃO AOS "IOGURTES" DE SOJA}

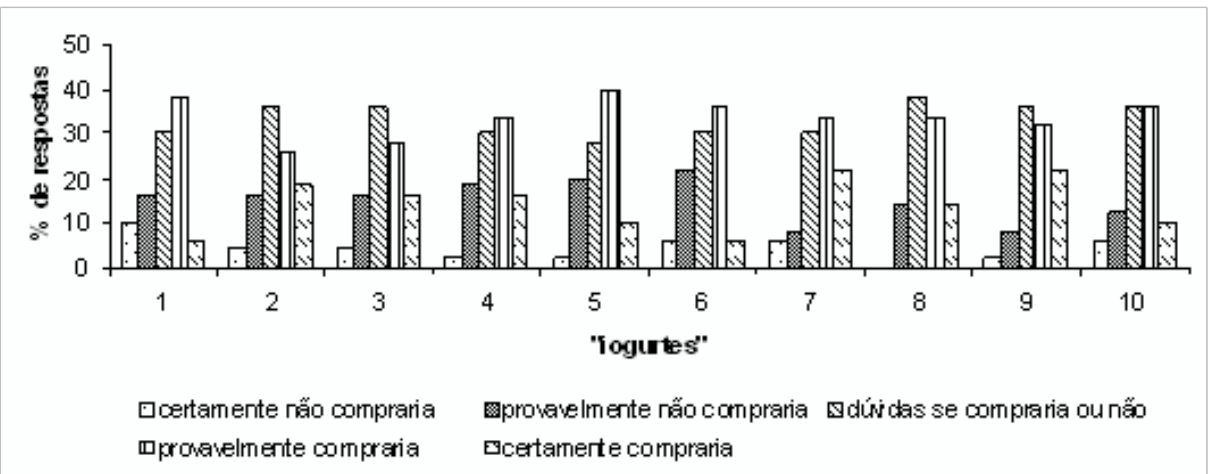

$1=0 \%$ Gelatina $(G)+0,5 \%$ Amido de Inhame $(A l)+0 \%$ Amidomax $4800^{\circledR}\left(A 4800^{\circledR}\right) ; 2=0 \%$ Gelatina $(G)+0 \%$ Amido de Inhame (Al) + 0,5\% Amidomax $4800^{\circledR}\left(A 4800^{\circledR}\right) ; 3=0 \%$ Gelatina $(\mathrm{G})+0,25 \%$ Amido de Inhame (Al) + 0,25\% Amidomax 4800 ${ }^{\circledR}\left(A 4800^{\circledR}\right)$; $4=0,2 \%$ Gelatina (G) + 0,3\% Amido de Inhame (Al) + 0\% Amidomax $4800^{\circledast}\left(A 4800^{\circledR}\right) ; 5=0,2 \%$ Gelatina (G) $+0 \%$ Amido de Inhame (Al) + 0,3\% Amidomax 4800 ${ }^{\circledR}\left(A 4800^{\circledR}\right) ; 6=0,3 \%$ Gelatina $(G)+0,2 \%$ Amido de Inhame (Al) + 0\% Amidomax $4800^{\circledR}$ $\left(A 4800^{\circledR}\right) ; 7=0,5 \%$ Gelatina (G) + 0\% Amido de Inhame (Al) + 0\% Amidomax 4800 ${ }^{\circledR}\left(A 4800^{\circledR}\right) ; 8=0,25 \%$ Gelatina (G) $+0,25 \%$ Amido de Inhame (AI) + 0\% Amidomax $4800^{\circledR}\left(A 4800^{\circledR}\right) ; 9=0,25 \%$ Gelatina $(\mathrm{G})+0 \%$ Amido de Inhame (Al) $+0,25 \%$ Amidomax $4800^{\circledast}\left(A 4800^{\circledR}\right) ; 10=0,15 \%$ Gelatina $(G)+0,2 \%$ Amido de Inhame $(A I)+0,15 \%$ Amidomax $4800^{\circledR}\left(A 4800^{\circledR}\right)$. 


\subsection{ANÁLISES FÍSICO-QUÍMICAS}

Na Tabela 3 são apresentados o tempo de fermentação e o pH final das amostras de "iogurte" de soja resultantes dos dez tratamentos em estudo.

\section{TABELA 3 - TEMPO DE FERMENTAÇÃO E pH FINAL DAS DEZ AMOSTRAS DE "IOGURTE" DE SOJA}

\begin{tabular}{ccc}
\hline Amostras & Tempo & pH final \\
\hline $\mathbf{1}$ & $6 \mathrm{~h} 20$ & 4,37 \\
$\mathbf{2}$ & $6 \mathrm{~h} 40$ & 4,36 \\
$\mathbf{3}$ & $6 \mathrm{~h} 40$ & 4,41 \\
$\mathbf{4}$ & $8 \mathrm{~h}$ & 4,43 \\
$\mathbf{5}$ & $8 \mathrm{~h} 20$ & 4,45 \\
$\mathbf{6}$ & $7 \mathrm{~h} 40$ & 4,40 \\
$\mathbf{7}$ & $8 \mathrm{~h}$ & 4,43 \\
$\mathbf{8}$ & $7 \mathrm{~h}$ & 4,38 \\
$\mathbf{9}$ & $8 \mathrm{~h}$ & 4,43 \\
$\mathbf{1 0}$ & $7 \mathrm{~h} 30$ & 4,42 \\
\hline
\end{tabular}

Médias seguidas pela mesma letra na mesma coluna não diferiram entre si $(p \leq 0,05)$.

$1=0 \%$ Gelatina + 0,5\% Amido de Inhame + 0\% Amidomax 4800 $; 2=0 \%$ Gelatina $+0 \%$ Amido de Inhame + 0,5\% Amidomax $4800^{\circledR} ; 3=0 \%$ Gelatina + 0,25\% Amido de Inhame + 0,25\% Amidomax $4800^{\circledR} ; 4=0,2 \%$ Gelatina + 0,3\% Amido de Inhame $+0 \%$ Amidomax $4800^{\circledast ;} ; 5=0,2 \%$ Gelatina $+0 \%$ Amido de Inhame + 0,3\% Amidomax $4800^{\oplus ;} 6=0,3 \%$ Gelatina + 0,2\% Amido de Inhame + 0\% Amidomax 4800 $; 7=0,5 \%$ Gelatina + 0\% Amido de Inhame + 0\% Amidomax 4800 ${ }^{\circledR} ; 8=0,25 \%$ Gelatina + 0,25\% Amido de Inhame + 0\% Amidomax 4800; $9=0,25 \%$ Gelatina + 0\% Amido de Inhame + 0,25\% Amidomax 4800 ${ }^{\oplus ;}$ $10=0,15 \%$ Gelatina $+0,2 \%$ Amido de Inhame $+0,15 \%$ Amidomax $4800^{\circledR}$.

As formulações contendo gelatina como espessante/estabilizante apresentaram maior tempo de fermentação, sugerindo que a gelatina interfere no processo de fermentação do "iogurte" de soja. É válido ressaltar que, sob o ponto de vista industrial, aumentos no tempo de fermentação se refletem no custo final dos produtos.

$\mathrm{Na}$ Tabela 4 são apresentados os valores médios de consistência, sinérese e capacidade de retenção de água das amostras de "iogurte" de soja.

Com relação à consistência, a amostra 7 com $0,5 \%$ de gelatina apresentou o melhor resultado e não diferiu significativamente $(p \leq 0,05)$ da amostra 2 com $0,5 \%$ de Amidomax $4800^{\circledast}$.

A amostra 7 também apresentou o melhor resultado para sinérese e não diferiu significativamente $(p \leq 0,05)$ da amostra 9 , com $0,25 \%$ de gelatina e $0,25 \%$ de Amidomax $4800^{\circledR}$. As demais amostras não apresentaram diferença significativa $(p \leq 0,05)$ entre si. Os resultados para o teste da capacidade de retenção de água foram coerentes com os da sinérese, evidenciando os melhores resultados para as amostras 7 e 9 .

ROSSI et al., 1984 observaram corpo inferior do "iogurte de soja" em relação aos dos iogurtes tradicionais, mesmo com a adição de sólidos de leite desengordurados e gelatina como espessante/ estabilizante nas concentrações máximas recomendadas (até 0,5\%). Observaram também que após sete dias de estocagem refrigerada, o produto apresentava nítidos sinais de sinérese. Nesse caso, a gelatina usada como espessante/estabilizante não apresentou os mesmos resultados físico-químicos observados no presente estudo, provavelmente em razão dos diferentes tipos de gelatina empregados. 
Em outro trabalho realizado por ROSSI et al. (1990), o "iogurte" de soja com gelatina a 0,25\% não apresentou bons resultados de consistência. Vale destacar que nesse caso os autores não testaram a concentração de $0,5 \%$ no produto.

\section{TABELA 4 - VALORES MÉDIOS DE CONSISTÊNCIA, SINÉRESE E CAPACIDADE DE RETENÇÃO DE ÁGUA DAS DEZ AMOSTRAS DE "IOGURTE" DE SOJA}

\begin{tabular}{cccc}
\hline Amostras & $\begin{array}{c}\text { Consistência } \\
(\mathbf{c m} / \mathbf{3 0 s})\end{array}$ & Sinérese (\%) & $\begin{array}{c}\text { Capacidade de } \\
\text { retenção de água } \\
(\%)\end{array}$ \\
\hline $\mathbf{1}$ & $14,50^{\mathrm{c}}$ & $17,43^{\mathrm{a}}$ & $34,43^{\mathrm{e}}$ \\
$\mathbf{2}$ & $10,40^{\mathrm{et}}$ & $17,31^{\mathrm{a}}$ & $37,74^{\mathrm{de}}$ \\
$\mathbf{3}$ & $11,50^{\mathrm{ed}}$ & $18,08^{\mathrm{a}}$ & $37,99^{\text {ad }}$ \\
$\mathbf{4}$ & $17,09^{\mathrm{ab}}$ & $19,01^{\mathrm{a}}$ & $37,55^{\mathrm{de}}$ \\
$\mathbf{5}$ & $14,86^{\mathrm{c}}$ & $19,26^{\mathrm{a}}$ & $37,98^{\text {ad }}$ \\
$\mathbf{6}$ & $16,56^{\mathrm{b}}$ & $13,96^{\mathrm{a}}$ & $38,44^{\text {ad }}$ \\
$\mathbf{7}$ & $9,93^{\mathrm{f}}$ & $0,06^{\mathrm{b}}$ & $71,67^{\mathrm{a}}$ \\
$\mathbf{8}$ & $18,23^{\mathrm{a}}$ & $15,91^{\mathrm{a}}$ & $36,54^{\mathrm{de}}$ \\
$\mathbf{9}$ & $14,66^{\mathrm{c}}$ & $0,00^{\mathrm{b}}$ & $45,56^{\mathrm{b}}$ \\
$\mathbf{1 0}$ & $12,70^{\mathrm{d}}$ & $13,19^{\mathrm{a}}$ & $41,31^{\mathrm{c}}$ \\
\hline
\end{tabular}

Médias seguidas pela mesma letra na mesma coluna não diferiram entre si $(p \leq 0,05)$.

$1=0 \%$ Gelatina $+0,5 \%$ Amido de Inhame $+0 \%$ Amidomax $4800^{\circledR} ; 2=0 \%$ Gelatina $+0 \%$ Amido de Inhame + 0,5\% Amidomax $4800^{\circledast} ; 3=0 \%$ Gelatina + 0,25\% Amido de Inhame + 0,25\% Amidomax $4800^{\circledR} ; 4=0,2 \%$ Gelatina + 0,3\% Amido de Inhame $+0 \%$ Amidomax $4800^{\circledR} ; 5=0,2 \%$ Gelatina $+0 \%$ Amido de Inhame + 0,3\% Amidomax $4800^{\circledR} ; 6=0,3 \%$ Gelatina + 0,2\% Amido de Inhame + 0\% Amidomax 4800 $7=0,5 \%$ Gelatina + 0\% Amido de Inhame + 0\% Amidomax 4800 ${ }^{\oplus}: 8=0,25 \%$ Gelatina + 0,25\% Amido de Inhame + 0\% Amidomax 4800 ${ }^{\oplus ;} 9$ = 0,25\% Gelatina + 0\% Amido de Inhame + 0,25\% Amidomax 4800 ${ }^{\oplus ;}$ $10=0,15 \%$ Gelatina $+0,2 \%$ Amido de Inhame $+0,15 \%$ Amidomax $4800^{\circledR}$.

JAWALEKAR et al. (1993), estudando formulações de iogurte a base de leite de vaca e de búfala e usando gelatina como espessante/estabilizante, demonstraram bons resultados para os testes consistência e sinérese.

KEOGH e O'KENNEDY (1998) estudaram as propriedades reológicas do iogurte com diferentes hidrocolóides. Verificaram que a gelatina contribuiu para o aumento de consistência do iogurte, mas que 0 amido de trigo não apresentou resultados satisfatórios. Os mesmos resultados foram observados para a sinérese.

Com a finalidade de melhor explorar as relações entre os atributos sensoriais e físico-químicos que caracterizam as dez amostras estudadas foi empregada a matriz de correlação de Pearson (Tabela 5).

SHIMAKURA e RIBEIRO JUNIOR (2005) classificaram a correlação em função do valor de $r$ da seguinte forma: para $\geq 0,90$ a correlação é considerada muito forte; entre 0,70 e 0,89 como forte; entre 0,40 e 0,69 como moderada; entre 0,20 e 0,39 como fraca e para valor $\leq 0,19$ é considerada muito fraca.

Pelos resultados da matriz de Pearson, ao nível de $5 \%$ de significância, foi verificada correlação forte $(r=0,81)$ entre os atributos impressão global e textura (ambos atributos sensoriais), enquanto que entre a impressão global e a capacidade de retenção de água obteve-se correlação moderada $(r=0,64)$. Esses resultados confirmam que firmeza adequada e boa capacidade de retenção de água contribuem muito para a aceitação do produto. A correlação negativa e forte $(r=-0,80)$ entre sinérese e capacidade de retenção de água deve-se ao método utilizado (em que as medidas são inversamente proporcionais). Isto é, o resultado de sinérese mostra a porcentagem de água liberada do produto enquanto a capacidade de retenção de água mostra a porcentagem de água retida no produto. 
TABELA 5 - COEFICIENTES DE CORRELAÇÃO DE PEARSON (r) ENTRE AS MÉDIAS DOS ATRIBUTOS SENSORIAIS

\begin{tabular}{|c|c|c|c|c|c|}
\hline & TEX & IG & CRA & SIN & CONS \\
\hline TEX & $\begin{array}{c}1 \\
(0,0)\end{array}$ & & & & \\
\hline IG & $\begin{array}{c}0,81 \\
(0,004)\end{array}$ & $\begin{array}{c}1 \\
(0,0)\end{array}$ & & & \\
\hline CRA & $\begin{array}{c}0,60 \\
(0,06)\end{array}$ & $\begin{array}{c}0,64 \\
(0,04)\end{array}$ & $\begin{array}{c}1 \\
(0,0)\end{array}$ & & \\
\hline SIN & $\begin{array}{c}-0,47 \\
(0,17)\end{array}$ & $\begin{array}{l}-0,55 \\
(0,10)\end{array}$ & $\begin{array}{c}-0,80 \\
(0,0055)\end{array}$ & $\begin{array}{c}1 \\
(0,0)\end{array}$ & \\
\hline CONS & $\begin{array}{c}-0,55 \\
(0,10)\end{array}$ & $\begin{array}{l}-0,36 \\
(0,30)\end{array}$ & $\begin{array}{c}-0,52 \\
(0,12)\end{array}$ & $\begin{array}{c}0,30 \\
(0,40)\end{array}$ & $\begin{array}{c}1 \\
(0,0)\end{array}$ \\
\hline
\end{tabular}

tex = textura; ig = impressão global; cra = capacidade de retenção de água; sin = sinérese; cons = consistência .

Números entre parêntesis representam o nível de significância de r.

\section{CONCLUSÃO}

Sob o ponto de vista sensorial, a gelatina utilizada de forma isolada como espessante/ estabilizante permitiu a obtenção de produto com boa aceitação e forte tendência de baixa rejeição. Vale ressaltar que a gelatina utilizada isoladamente e também em associação aos outros espessantes/ estabilizantes provocou aumento no tempo de fermentação do "iogurte" de soja.

Os produtos processados apenas com a gelatina apresentaram maior consistência, menor sinérese e maior capacidade de retenção de água.

Foi possível verificar a existência de correlação entre a aceitação dos produtos em termos de impressão global e parâmetros que determinam a textura como, por exemplo, a consistência, a capacidade de retenção de água e a sinérese.

\section{ABSTRACT \\ SENSORIAL AND PHYSICAL-CHEMICAL ASPECTS OF SOY "YOGURTS" WITH YAM (Dioscorea Alata) STARCH, MODIFIED STARCH AND GELATIN AS STABILIZERS/THICKENERS}

This study had as objective to evaluate the effect of yam starch, modified starch from Cargill-Brasil (Amidomax $4800^{\circledR}$ ) and gelatin from Gelita-Brasil (GEL-LAC) as stabilizers/thickeners in different ratios and combinations in the soy "yoghurt" fermented with Enterococcus faecium and Lactobacillus helveticus ssp jugurti. Ten soy "yoghurt" formulations containing these different stabilizers/thickeners, always totalizing $0.5 \%$ in relation to the final formulation, were analyzed in sensorial and physical-chemical terms. Based on the observed results, it was concluded in relation to the sensorial point of view that the more appropriate product was processed only with gelatin at $0.5 \%$ concentration. This product also presented the best physical-chemical results related to consistency, syneresis and water holding capacity. However, the isolated use of gelatin increased fermentation time of the soy "yoghurt".

KEY-WORDS: SOY "YOGHURT"; YAM; MODIFIED STARCH; GELATIN; SENSORIAL ANALYSIS; PHYSICALCHEMICAL ANALYSIS.

\section{REFERÊNCIAS}

1 AOAC. Association of Official Analytical Chemists. Official methods of analisys of the AOAC International. $16^{\text {th }}$ ed. Washington, D.C, 1996.

2 BEDANI, R. Efeito do consumo de "iogurte" de soja suplementado com isoflavonas e cálcio sobre o tecido ósseo de ratas maduras ovariectomizadas. Araraquara, 2005. 101 f. Dissertação (Mestrado em Ciência dos Alimentos), Faculdade de Ciências Farmacêuticas, Universidade Estadual Paulista.

3 BOSTWICK CONSISTOMETER. Operating instructions. Fairfax (VA): CSC Scientific Company, [s.d.]. 
4 CEREDA, M.P. (Coord.). Culturas de tubersosas amiláceas latino americanas: propriedades gerais do amido. Campinas: Fundação Cargill, 2001. v.1.

5 DAIUTO, E.R. Características de féculas de tuberosas e suas relações com resistência dos géis sob condições de estresse aplicada na industrialização de alimentos. Botucatu, 2005.146 p. Tese (Doutorado em Agronomia/Energia na Agricultura) - Faculdade de Ciência Agronômicas, Universidade Estadual Paulista.

6 DAIUTO, E.R.; CEREDA, M.P. Extração de fécula de inhame (Dioscorea sp.). In: CEREDA, M.P.; VILPOUX, O.F. (Coord). Tecnologia, usos e potencialidades de tuberosas amiláceas Sul Americanas. São Paulo: Fundação Cargill, 2003. p.176-190.

7 GUERREIRO, L.M.R. Avaliação de amidos em condições de estresse adaptados ao processamento de alimentos. Botucatu, 2002. 180 p. Dissertação (Mestrado em Agronomia/Energia na Agricultura) - Faculdade de Ciência Agronômicas, Universidade Estadual Paulista.

8 HASSAN, A.N.; FRANK, J.F.; SCHMIDT, K.A.; SHALABI, S.I. Textural proprieties of yogurt made with encapsulated nonropy lactic cultures. J. Dairy Sci., v. 79, n.12, p. 2098-2103, 1996.

9 HARTE, F.; LUEDECKE, L.; SWANSON, B.; BARBOSA-CÁNOVAS, G.V. Low fat set yogurt made from milk subjected to combinations of high hydrostatic pressure and thermal processing. J. Dairy Sci., v. 86, n. 4, p. 1074-1082, 2003.

10 JAWALEKAR, S. D.; INGLE, U. M.; WAGHMARE, P. SS.; ZANJAD, P. N. Influence of hydrocolloids on rheological and sensory properties of cow and buffalo's yoghurt. Indian J. Dairy Sci., v. 63, n.1, p. 217-219, 1993.

11 KEOGH, M. K.; O'KENNEDY, B. T. Rheology of stirred yoghurt as affected by added milk fat, protein and hydrocolloids. J. Food Sci.. v. 63, n.1, p. 108-112, 1998.

12 KUMAR, P.; MISHRA, H. N. Mango soy fortified set yoghurt: effect of stabilizer addition on physicochemical, sensory and textural properties. Food Chem., v. 87, p. 501-507, 2004.

13 MALI, S.; FERRERO, C.; REDIGONDA, V.; BALEIA, A. P.; GROSSMANN, M. V. E.; ZARITZKY, N. E. Influence of pH and hydrocolloids addition on yam (Dioscorea alata) starch pastes stability. Lebensm.-Wiss. u-Technol., v. 36, p. 475481, 2003.

14 MORAES, M. A. C. Métodos para avaliação sensorial dos alimentos. Campinas: UNICAMP, 1985.85 p.

15 ROSSI, E.A.; REDDY, K.V.; SILVA, R.S.S.F. Formulation of soy-whey yogurt, using response surface methodology. Arq. Biol. Tecnol., v.27, p.387-390, 1984.

16 ROSSI, E.A.; FARIA, J.B.; BORSATO, D.; BALDOCHI, F.L. Otimização de um sistema estabilizante para o "iogurte" de soja. Alim. Nutr., São Paulo, v.2, p.83-92, 1990.

17 ROSSI, E. A.; VENDRAMINI, R. C.; CARLOS, I. Z.; UEIJI, I. S.; SQUINZARI JR, M. M.; SILVA, I. S.; VALDEZ, G. F. Efeito de um novo produto fermentado de soja sobre os lipídeos séricos de coelhos hipercolesterolêmicos. Arq. Bras. Cardiol., v. 74, n.3, p. 209-212, 2000.

18 ROSSI, E. A.; VENDRAMINI, R. C.; CARLOS, I. Z.; OLIVEIRA, M. G.; VALDEZ, G. F. Efeito de um novo produto fermentado de soja sobre os lípides séricos de homens adultos normocolesterolêmicos. Arch. Latinoamer. de Nutr., v. 53, n. 1, p.47-51, 2003.

19 SAS Institute. Statistical analytical system. Cary, 1996. Version 6.12.

20 Shiguemoto, G. E.; ElizeU, E. A.; BAldisserA, V.; GOUVEiA, C. H.; VARGAS, G. M. F. V.; PEREZ, S. E. A. Isoflavone-supplemented soy yoghurt associated with resistive physical exercise increase bone mineral density of ovariectomized rats. Maturitas, v. 57, p. 261-270,2007.

21 SHIMAKURA, S. E.; RIBEIRO JUNIOR, P. J. Estatística. Disponível em: <http://www.est.ufpr.br/ paulojus/CE003/ ce003/> Acesso em: 05 dez. 2006.

22 STONE, H.; SIDEL, J.L. Sensory evaluation practices. $2^{\text {nd }}$ ed. London: Academic Press, 1993.338 p.

23 TAMIME, A.Y.; ROBINSON, R.K. Yoghurt: science and technology. Oxford: Pergamon, 1985.431 p.

24 VENDRAMINI, R. C. Efeito da ingestão de um produto de soja fermentado com Enterococcus faecium e Lactobacillus helveticus na produção de citocinas, óxido nítrico e peróxido de hidrogênio. Araraquara, 2002. 96 f. Dissertação (Mestrado em Análises Clínicas), Faculdade de Ciências Farmacêuticas, Universidade Estadual Paulista. 